> Bien des années après avoir découvert que, à l'instar des neurones, les cellules du système immunitaire communiquent entre elles au niveau de jonctions spécialisées de type synapse, la fonction de ces structures est ainsi toujours discutée. La raison de ce questionnement persistant est sans doute à rechercher dans une surinterprétation de l'organisation moléculaire particulière que l'on observe dans des conditions expérimentales peu physiologiques à la jonction cellule T/cellule présentatrice d'antigène, et dans une généralisation abusive du modèle qui en a été tiré. L'ambition de cet article est donc de faire le point sur les limites d'une vision provisoirement dominante de la synapse immunologique, et de présenter un autre point de vue sur cette structure et son fonctionnement. <

\title{
La synapse immunologique : des modèles aux réalités
}

\author{
Georges Bismuth, Alain Trautmann
}

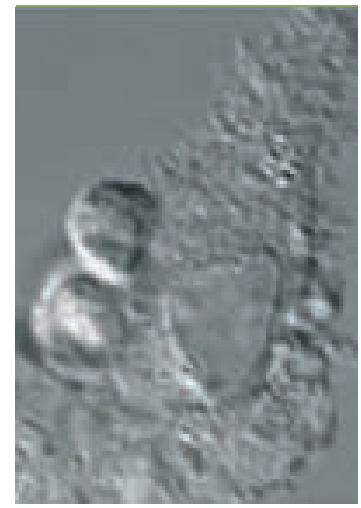

cific protein tyrosine kinase) et Zap70 ( $\zeta$ chain (TCR) associated protein kinase $70 k D a$ ), et la protéine kinase $C \theta$. De là l'établissement d'un paradigme, résumant cette structure à deux régions principales concentriques : centrale, ou c-SMAC (central supramolecular activation cluster), correspondant théoriquement à une zone d'apposition serrée entre les deux membranes, compte tenu de la taille des molécules engagées dans cette zone, et périphérique, ou p-SMAC (peripheral supramolecular activation cluster), où la distance prédite entre les membranes pré- et postsynaptiques devrait être environ 3 fois plus grande qu'au centre. De là aussi l'idée, vite dominante, que cette structure synaptique précise était indispensable au déclenchement de l'activation $T$ en ajustant correctement dans le plan de la membrane lymphocytaire les différents acteurs moléculaires de la réponse [3]. On verra que ce raisonnement s'appliquait mieux au modèle qu'aux données expérimentales réelles. En l'absence de données sur la taille de la fente synaptique, cette vision schématique était en effet construite sur des observations effectuées dans des conditions expérimentales particulières (APC artificielles, comme des bicouches lipidiques ou des lymphomes $B$, dans tous les cas peu ou pas mobiles, et présentant toujours des quantités très importantes, non physiologiques, d'antigène) et qui, trop vite, ont été constituées en norme. De plus, ce type d'agencement synaptique stable, quand il se produit, n'est observé qu'après

Article reçu le 17 février 2006, accepté le 14 avril 2006. 
des temps d'interaction relativement longs (plusieurs minutes à quelques dizaines de minutes) après le début du contact. Ainsi, sans remettre en cause le rôle de la SI comme un lieu privilégié d'échange d'informations entre cellules du système immunitaire, l'ensemble de ces éléments justifie un réexamen de sa pertinence physiologique.

Avant cette analyse critique, il convient de préciser ici l'objet dont on parle, car il n'y a pas une, mais de nombreuses SI, par exemple celles formées entre cellules T et cellules B, entre cellules NK (natural killer)

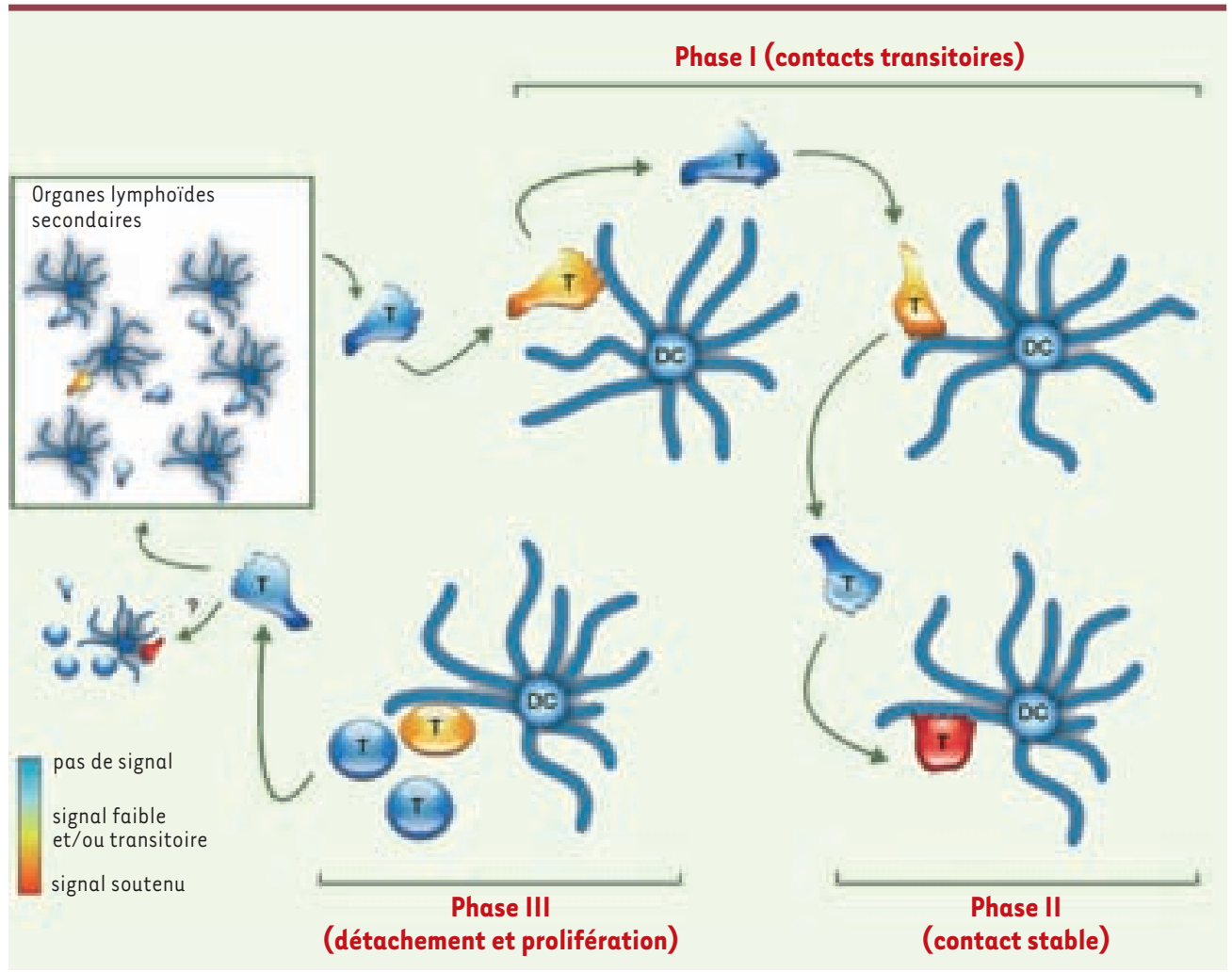

et leur cibles, etc. Les partenaires moléculaires étant différents dans chaque cas, chacun de ces objets doit donc être analysé spécifiquement, et il est important de se garder de généralisations anticipées. Dans ce qui suit, nous porterons un intérêt particulier à la structure et au fonctionnement des synapses formées par des cellules T naïves, en particulier avec les APC naturelles que sont les cellules dendritiques (DC). Rappelons que si d'autres cellules comme les cellules B ou les macrophages peuvent également présenter des antigènes à des cellules $T$ activées, les DC sont les seules APC capables, in vivo, d'activer pleinement des cellules T naïves.

\section{Synapses stables et instables}

On sait maintenant que la reconnaissance de l'antigène à la surface d'une DC par un lymphocyte $T$ naïf passe par différentes phases (Figure 1). La première se traduit par des déformations et une scrutation systématique (scanning) de l'APC, mettant en jeu des chimiokines et des molécules d'adhésion comme LFA-1.

Figure 1. Contacts stables et instables. Les lymphocytes T circulent en permanence au sein des organes lymphoïdes secondaires. De nombreuses collisions avec les cellules dendritiques (DC) se produisent, qui peuvent déclencher, en l'absence d'antigène, de petites réponses transitoires. En présence d'antigène trois grandes phases sont observables. Phase I: pendant quelques heures, les cellules T naïves ont des interactions transitoires de quelques minutes avec les DC présentatrices. On ignore comment s'organise la zone de contact à ce stade. II est très probable que des signaux d'activation efficaces sont délivrés durant cette phase, comme en témoigne l'apparition de marqueurs d'activation (comme le CD69) et la stimulation de la transcription de certains gènes (comme celui de l'interleukine 2). Cette phase n'est en général pas observée in vitro, sauf cas particulier dans des matrices de collagène. Si l'on imagine la stabilité de l'interaction T-DC comme la résultante d'un ensemble de forces favorisant ou non l'interaction, cela suggère que la pression de l'environnement pour une mobilité importante des cellules T (la même sans doute que celle qui est en permanence à l'œuvre pour provoquer leur mobilité de base) est encore dominante à ce stade. On conçoit aisément que la quantité d'antigène présenté par les $D C$ et l'affinité du récepteur T pour ce dernier soit un paramètre clef pour moduler la durée de cette première phase. Phase II : il s'agit d'une phase de contact très prolongé (jusqu'à 24h) et stable. Une hypothèse pour l'expliquer serait que les contacts itératifs de la phase précédente modifient l'affinité ou l'expression de molécules d'adhésion. Une baisse de sensibilité des cellules T au signal «mouvement» est également possible, par exemple par une modulation négative de certains récepteurs des chimiokines. Une zone de contact étendue entre la cellule T et la DC est formée à ce stade, au sein de laquelle, par analogie avec ce qui a été observé in vitro (mais jamais analysé in vivo), des zones d'apposition multiples et dynamiques véhiculeraient le signal soutenu observé durant cette phase et nécessaire à l'entrée en cycle des cellules T. Phase III : cette phase est encore mal connue, notamment en ce qui concerne la nature du contrôle qui entraîne la fin du signal et le détachement des cellules T. On ignore également si les mitoses multiples qui affectent un lymphocyte T s'effectuent au contact de la première DC stimulatrice, sinon dans son environnement proche, ou avec d'autres DC, après que les cellules aient retrouvé leur mobilité initiale. 
En réalité, cette phase est indépendante de la présence de l'antigène et reflète les mécanismes contrôlant les déplacements de tous les lymphocytes $T$ au sein des tissus lymphoïdes. Après engagement du TCR, on observe une réduction de la mobilité de la cellule T. L'interaction T-DC peut ensuite durer plusieurs heures avant de se rompre, juste avant la division cellulaire. Cette séquence est observable in vitro mais, surtout, sa réalité physiologique a récemment été confirmée et précisée in vivo par des études effectuées directement au sein des organes lymphoïdes, par microscopie biphotonique [4-8].

Cette séquence dynamique d'événements complexes, au cours desquels l'existence de structures synaptiques prototypiques n'a jamais été démontrée, s'accommode mal du modèle simplifié, et encore dominant, dans lequel on pourrait distinguer des synapses «matures», prototypiques, efficaces, et des synapses «immatures », encore incomplètement structurées, et inefficaces. Cette opposition binaire est peu fondée, car on sait que le déclenchement de l'activation T et des réponses précoces en aval du TCR, comme l'accumulation de phosphotyrosines sous l'action des kinases Lck et ZAP70 , ou encore la réponse calcique, interviennent en quelques secondes, alors que le contact entre cellules $T$ et $A P C$ est très ténu, sans aucun signe d'une organisation moléculaire fortement structurée [9].

\section{Facteurs facilitant la détection de l'antigène}

De récents travaux suggèrent l'existence d'une certaine forme de préorganisation de récepteurs membranaires. Ainsi, le TCR peut se présenter sous forme multivalente avant toute activation [10]. Il est possible qu'il en soit de même pour des molécules de signalisation, et que ces éléments préassemblés puissent favoriser le déclenchement de la réponse T. Il est également envisageable que, lors de la première phase de scanning des APC par les cellules $T$, des contacts instables puissent jouer un véritable rôle de sensibilisation des cellules $T$, comme nous l'avons récemment montré [11]. Dans le même ordre d'idées, des chimiokines telles que CCL19 et CCL21, très vraisemblablement impliquées dans ce processus de scanning, pourraient jouer un rôle supplémentaire: de fait, leur addition à des cellules T naïves, qui expriment leur récepteur, CCR7, aboutit à une amplification de la réponse à la stimulation du TCR [12]. La libération de ces chimiokines par l'APC, agissant localement dès que le conjugué commence à se former (et sans doute aux stades plus tardifs), pourrait également leur permettre de jouer un rôle de costimulation directe [13], donc de vecteurs solubles d'information à la synapse ; ces chi- miokines pourraient alors être considérées comme des «immunotransmetteurs» [14].

\section{Synapses organisées de façon visible ou non}

La critique d'une vision figée d'une synapse «mature » porte sur sa dynamique, mais aussi sur son organisation spatiale. En effet, la SI concentrique est le plus souvent observée lorsque l'on emploie des APC qui sont pratiques d'un point de vue expérimental, mais non physiologiques (lignée $B$, bicouche lipidique dans laquelle ont été insérés les ligands du TCR ou de LFA-1). Même avec ces APC, cette structure n'est observée que pour des concentrations d'antigène très élevées, physiologiquement improbables, et ne l'est pas avec les DC. Des études réalisées dans notre laboratoire, combinant immunofluorescence dynamique et analyse quantitative en microscopie électronique, ont en effet montré que cette synapse T-DC présente une structure multi-

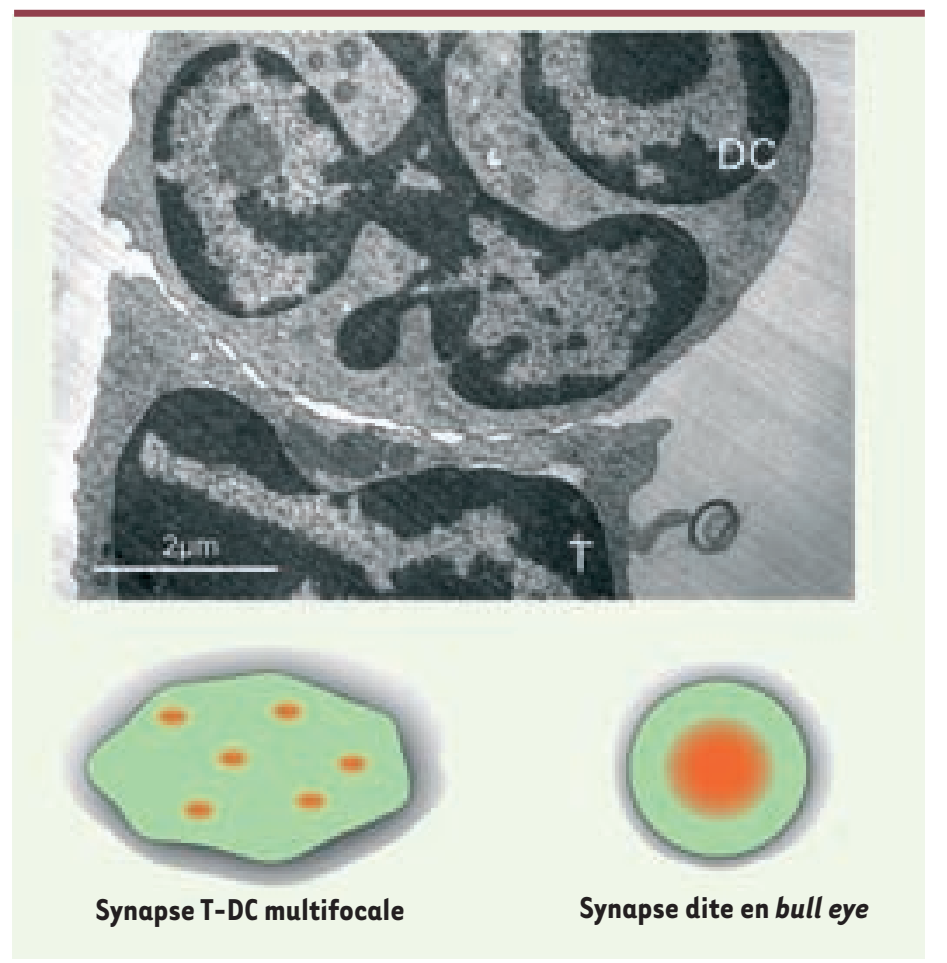

Figure 2. La synapse T-DC est multifocale. Image en microscopie électronique d'une synapse immunologique (SI) formée entre un lymphocyte $T$ naïf murin et une DC splénique autologue, en présence de l'antigène. On peut voir à la fois des zones d'apposition très serrées et des zones d'apposition beaucoup plus lâches, montrant le caractère peu organisé dans son ensemble de la zone d'interaction, pourtant très étendue, formée par les deux cellules. Chacune de ces zones très serrées pourrait agir comme une unité de signalisation indépendante (une «microsynapse »), dont la durée de vie pourrait être brève, et remplacée par d'autres pendant toute la durée du contact. On aurait ainsi un système extrêmement fluctuant et dynamique, multifocal, très éloigné du modèle de SI concentrique en bull eye observé avec des APC (cellules présentatrices d'antigène) non conventionnelles (Cliché Graça Raposo, Institut Curie, Paris, France). 
focale dans laquelle des zones d'apposition étroite, sur quelques dizaines de nanomètres chacune, alternent avec des zones d'apposition lâche des deux membranes, l'ensemble fluctuant vraisemblablement très rapidement au sein de la synapse [15]. La vision d'une synapse figée et concentrique doit, dans ce cas, laisser la place à celle d'une structure fluide et multifocale (Figure 2).

Pourquoi les observations faites sur des synapses T-DC n'entrentelles pas dans le cadre du modèle établi auparavant? Pour expliquer la spécificité de la synapse T-DC, certains ont cru pouvoir impliquer DC-SIGN (dendritic cell-specific intercellular adhesion molecule-3 (ICAM-3) grabbing nonintegrin), une molécule exprimée très préférentiellement sur les $D C$; nous avons toutefois montré que cette explication était incorrecte [16]. Une partie de l'explication pourrait être liée à l'activité élevée du cytosquelette d'actine des DC, sous le contrôle de petites protéines $G$ de la famille Rho $[17,18]$, qui con-

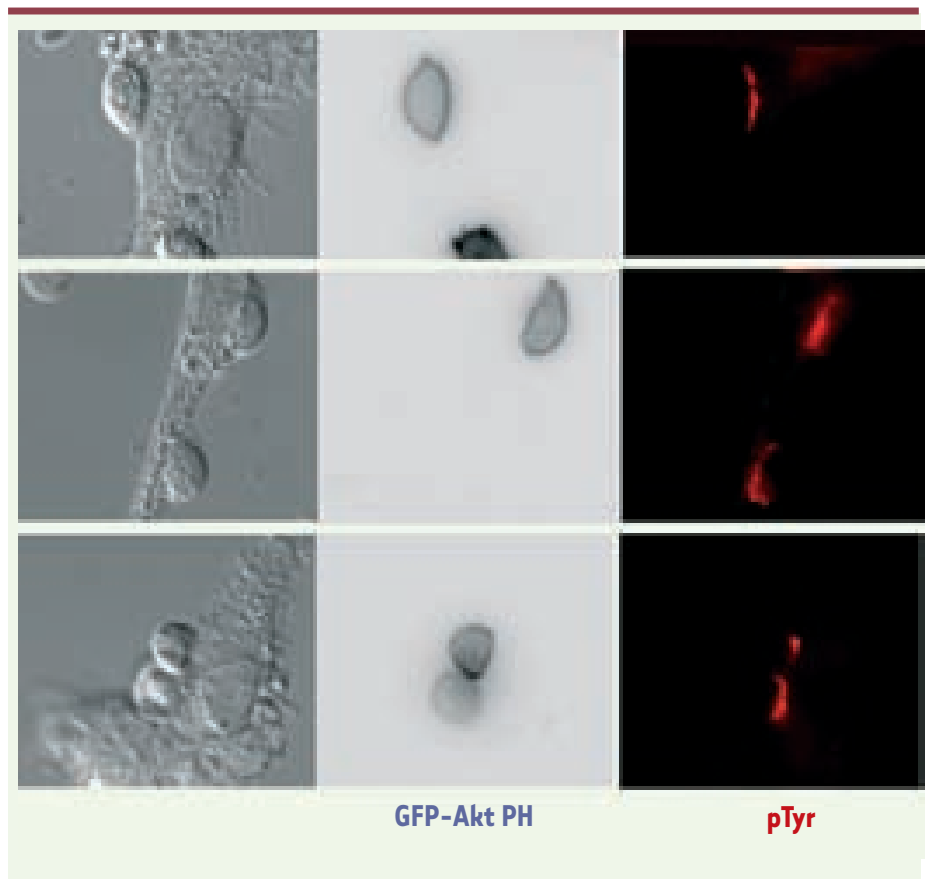

Figure 3. Activation soutenue à la synapse immunologique T-DC. Images montrant l'activation de la PI3K et l'accumulation synaptique de phosphotyrosines ( $p$ Tyr) dans des conjugués formés entre des lymphocytes T CD4 ${ }^{+}$naïfs humains et des $D C$ matures 4 heures après le début du contact. L'activation de la voie de la PI3K est mise en évidence par l'accumulation membranaire d'une sonde fluorescente, la molécule Akt PH couplée à la green fluorescent protein (GFP-Akt PH), qui se relocalise sur les produits de cette voie métabolique (les 3'-phosphoinositides) à la membrane plasmique. La présence de la sonde dans l'ensemble de la membrane est liée à la diffusion rapide des 3'-phospho-inositides depuis la $\mathrm{SI}$, où ils sont produits grâce au recrutement des sous-unités régulatrices de la classe la des PI3K, via des interactions pTyr-SH2 (voir [26]). Dans ce système, le maintien sur des temps prolongés d'un niveau élevé de phosphotyrosines à la SI et la persistance de l'activation de cette voie métabolique, qui joue un rôle clé dans la prolifération cellulaire, sont strictement dépendants. duit ces cellules à présenter des phénomènes actifs de projection/rétraction de dendrites et de déformations intenses de la membrane plasmique que l'on observe très peu avec d'autres APC comme les cellules B (et, bien sûr, pas du tout avec une bicouche lipidique). Par ailleurs, les DC matures sécrètent de nombreux facteurs solubles, chimiokines et cytokines [IL-8 (interleukine 8), MIPI $\beta$ (macrophage inflammatory protein $1 \beta$ ), Rantes (regulated upon activation, normal T-cell expressed and secreted), TARC (thymus and activation-regulated chemokine)...], susceptibles d'agir sur les cellules T, leur mobilité ou la fluidité de leur membrane.

En outre, même avec des APC comme les cellules B, susceptibles de former des synapses concentriques, la notion de synapse mature stable doit être remise en cause. De récents travaux auxquels nous avons collaboré montrent ainsi qu'un lymphocyte $T$ peut rapidement réorganiser les molécules présentes dans une $\mathrm{SI}$ mature, déjà formée avec une première $A P C$, vers une autre APC présentant des concentrations d'antigène plus élevées [19].

En conclusion, le caractère statique et figé du modèle conventionnel de SI mature, qui n'a jamais été établi in vivo, semble de moins en moins compatible avec les données les plus récentes obtenues in vitro.

\section{Les microclusters, une organisation supramoléculaire élémentaire?}

En ce qui concerne la présence d'une structuration synaptique supramoléculaire, des travaux récents employant le système de bicouche lipidique ont montré l'existence, dès le début du contact, de microclusters périphériques contenant le TCR et les kinases ZAP-70 et Lck. Ces microclusters nouvellement formés sont le siège de phénomènes de signalisation, comme en témoignent l'abondance locale de phosphotyrosines et le recrutement des adaptateurs LAT et SLP-76 (SH2 domain-containing leukocyte protein of $76 \mathrm{kDa})[20,21]$. Ces structures migrent en permanence vers le centre pour former le c-SMAC enrichi en TCR dans lequel, curieusement, les phosphorylations disparaissent dans une sorte de «trou noir » au niveau duquel le TCR serait internalisé. Ces observations aboutissent à suggérer que, dans ce système expérimental, l'unité de signalisation serait le microcluster. De telles structures n'ont pas été mises en évidence par les mêmes méthodes dans des synapses T-DC, mais leur abondance et leur taille sont parfaitement compatibles avec les zones d'apposition étroite observées dans celles-ci. Les signaux initiaux pourraient donc être délivrés au niveau de ces «signalosomes » élémentaires, éventuellement en partie 
préformés. Cela expliquerait l'efficacité remarquable d'une structure où quelques molécules de TCR suffisent à déclencher une réponse, qui démarre bien avant que la zone de contact T-APC ait fini de se mettre en place [22]. Soulignons que, in vivo, en présence d'antigène à la surface des DC, les collisions aléatoires T-DC donnent lieu pendant les premières heures à des interactions transitoires et répétées, avant que les lymphocytes ne s'arrêtent au contact de l'une d'entre elles [4]. II est clair que des signaux d'activation (dont la nature et les modes de déclenchement restent mystérieux) sont délivrés lors de cette phase, et mettent en jeu des synapses nécessairement peu structurées.

\section{L'activation soutenue à travers la SI : une étape nécessaire pour sortir les cellules T de leur état de quiescence}

Que savons-nous aujourd'hui de la persistance de la signalisation synaptique? Des travaux initiaux avaient conclu que ces signaux, en particulier l'activation des protéine tyrosine kinases, étaient transitoires, agissant comme de simples facteurs déclenchants [9]. Des signaux off précoces stopperaient ainsi l'activation, leur existence constituant une des fonctions principales de la SI. Ces processus de contrôle de la fin du signal existent évidemment. Ils mettent en jeu l'internalisation et la dégradation du complexe TCR/CD3 et de ses relais de signalisation, et le recrutement de molécules inhibitrices, comme la phosphatase SHP-1 (Src homology region 2 domain-containing phosphatase 1) ou l'adaptateur CD2-AP (CD2 associated protein) [23]. En revanche, il est étrange, paradoxal et d'ailleurs peu fondé (même si cela a été beaucoup répété) de proposer qu'une structure de communication entre deux cellules serve principalement à transmettre... l'arrêt de la communication.

On sait maintenant que lors d'une interaction T-APC prolongée, la cellule $T$ reçoit des signaux de façon soutenue (et sans doute $y$ « répond ») longtemps après la réponse initiale. Ainsi, l'augmentation de la concentration en calcium intracellulaire dans la cellule $T$ est généralement prolongée; de même, la voie de la phosphatidylinositol 3-kinase (PI3K), qui dépend d'une accumulation prolongée de phosphotyrosines dans la $\mathrm{SI}$, est encore active plusieurs heures après le début de l'interaction T-APC (Figure 3) [24-26]. Quels sont les acteurs moléculaires de ce signal soutenu? II est vraisemblable que cela dépende à la fois du TCR (qui donne la spécificité du signal), avec un réamorçage continu de sa reconnaissance à la SI grâce à des mécanismes de recyclage [27], et de l'engagement accru de molé- cules de costimulation (qui fournissent l'amplification indispensable). Expérimentalement, l'interruption du signal dépendant du TCR (par blocage des complexes CMH-peptide sur un conjugué préformé) arrête d'ailleurs rapidement ces signaux soutenus [28].

Une conséquence majeure de l'activation $T$ est la division cellulaire et l'expansion clonale, qui exigent la régulation transcriptionnelle de nombreux gènes, régulation que des signaux rapidement interrompus ne pourraient pas permettre. On peut y voir une des raisons du signal soutenu, raison peu prise en compte par ceux qui imaginaient un signal initial rapidement interrompu. Nous avons pu préciser ces notions dans de récents travaux réalisés sur les facteurs transcriptionnels de la famille Fox0 (forkhead transcription factors). Ces facteurs, lorsqu'ils sont nucléaires, contribuent à maintenir les cellules $T$ dans un état quiescent, en exerçant un contrôle permanent sur les niveaux d'expression de puissants régulateurs négatifs du cycle cellulaire, comme p27. Cet effet répresseur est levé après activation de la PI3K, via un de ses effecteurs, la sérine-thréonine kinase Akt, qui phosphoryle les Fox0 et provoque leur exclusion du noyau [26]. Ce phénomène, observable dès les premières minutes après le début du contact, persiste ensuite pendant plusieurs heures, et son blocage, même très tardif, empêche la prolifération des lymphocytes T. Cela constitue une des premières analyses fonctionnelles précises de signaux soutenus délivrés au niveau de la SI, et nécessaires à la prolifération T.

\section{Conclusions}

Si la synapse «mature», concentrique, statique et associée à une fonction simple reste présente à bien des esprits, cette description est vouée à disparaître. Elle correspond à une vision réductrice, figée, qui ne prend en compte ni la diversité des synapses immunologiques ou leur caractère fluide et dynamique, ni la multiplicité des signaux échangés entre les deux partenaires synaptiques, qui varient en fonction du temps. La volonté de simplification et la surinterprétation de données obtenues avec un dispositif expérimental commode, mais éloigné de la réalité, ont contribué à cette vision déformée. L'exploitation de données obtenues avec des systèmes physiologiques devrait permettre de corriger cette conception. Le retour de la physiologie, pour cette question, a d'abord signifié l'analyse de synapses T-DC in vitro, puis la prise en compte de données obtenues in vivo, dans l'environnement ganglionnaire, certes complexe et difficile à manipuler, mais qui évite les simplifications abusives. La microscopie biphotonique a déjà commencé et continuera à être un des outils privilégiés de ce type d'exploration de la vie des lymphocytes. $\diamond$

\section{SUMMARY}

The immunological synapse: models facing facts

The notion of immunological synapse is generally associated to a concentric structure (a core of T cell receptors surrounded by a ring of adhesion molecules) often called "mature synapse". This schematic view has been built on observations corresponding to peculiar experimental conditions: very high antigen concentration presented by surrogate APCs such as lipid bilayers or B lymphoma. These observations have been hastily constituted in a dogma that 
a "normal" synapse should look like this, should form only in the presence of antigen, and should trigger a "stop" signal that completely immobilizes the T cell. However, when analyzing the interaction between naive T cells and dendritic cells (DC), that are the only antigen-presenting cells able to activate naive $T$ cells, a very different picture emerges. Firstly, T-DC synapses can form in the absence of antigen; therefore antigen recognition is not a prerequisite for synapse formation. Secondly, these antigen-independent synapses are likely to play several roles, including sensitization of T cells for later antigen detection, and delivery of survival signals. Thirdly, in vivo, naive T cells interacting with antigen-laden $D C$ do not fully stop, but start to make transient contacts with DCs for a few minutes, before continuing their exploration. It is only after several hours of this process that T cells eventually immobilize. Fourthly, the structure of the T-DC synapse is clearly multifocal, the two cells interacting through several tens of tight appositions of a few tens of $\mathrm{nm}$ in diameter. These numerous tight appositions are reminiscent of the microclusters that have been recently described at the T-bilayer interface. Finally, synaptic signaling is not a transient initial event, but is sustained for hours. In particular, sustained activation of phosphatidylinositol 3-kinase allows the exclusion out of the nucleus of Fox 0 transcription factors, normally maintaining $T$ cells in a quiescent state. $\diamond$

\section{RÉFÉRENCES}

1. Davis DM, Dustin ML. What is the importance of the immunological synapse? Trends Immunol $2004 ; 25: 323-7$.

2. Monks CR, Freiberg BA, Kupfer $\mathrm{H}$, et al. Three-dimensional segregation of supramolecular activation clusters in T cells. Nature $1998 ; 395: 82-6$.

3. Grakoui A, Bromley SK, Sumen C, et al. The immunological synapse : a molecular machine controlling T cell activation. Science $1999 ; 285: 221-7$.

4. Mempel TR, Henrickson SE, Von Andrian UH. T-cell priming by dendritic cells in lymph nodes occurs in three distinct phases. Nature $2004 ; 427$ : 154-9.

5. Miller MJ, Safrina 0, Parker I, Cahalan MD. Imaging the single cell dynamics of CD4 ${ }^{+}$T cell activation by dendritic cells in lymph nodes. J Exp Med $2004 ; 200: 847-56$.

6. Miller MJ, Wei SH, Parker I, Cahalan MD. Two-photon imaging of lymphocyte motility and antigen response in intact lymph node. Science $2002 ; 296: 1869-73$.

7. Bousso $P$, Robey $\varepsilon$. Dynamics of $C D 8^{+} T$ cell priming by dendritic cells in intact lymph nodes. Nat Immunol $2003 ; 4: 579-85$.

8. Stoll S, Delon J, Brotz TM, Germain RN. Dynamic imaging of T cell-dendritic cell interactions in lymph nodes. Science $2002 ; 296$ : 1873-6.

9. Lee $\mathrm{KH}$, Holdorf $\mathrm{AD}$, Dustin $\mathrm{ML}$, et al. T cell receptor signaling precedes immunological synapse formation. Science $2002 ; 295$ : 1539-42.

10. Schamel WW, Arechaga I, Risueno RM, et al. Coexistence of multivalent and monovalent TCRs explains high sensitivity and wide range of response. J Exp Med $2005 ; 202$ : 493-503.

11. Randriamampita C, Boulla G, Revy P, et al. T cell adhesion lowers the threshold for antigen detection. Eur J Immunol $2003 ; 33: 1215-23$.
12. Kaiser A, Donnadieu E, Abastado JP, et al. CC chemokine ligand 19 secreted by mature dendritic cells increases naive T cell scanning behavior and their response to rare cognate antigen. J Immunol 2005 ; $175: 2349-56$.

13. Molon B, Gri G, Bettella M, et al. T cell costimulation by chemokine receptors. Nat Immunol $2005 ; 6: 465-71$.

14. Trautmann A. Chemokines as immunotransmitters? Nat Immunol 2005 ; $6: 427-8$.

15. Brossard C, Feuillet V, Schmitt A, et al. Multifocal structure of the T celldendritic cell synapse. Eur J Immunol 2005 ; 35 : 1741-53.

16. Real $\varepsilon$, Kaiser $A$, Raposo $G$, et al. Immature dendritic cells (DCs) use chemokines and intercellular adhesion molecule (ICAM)-1, but not DC-specific ICAM-3-grabbing nonintegrin, to stimulate CD4 ${ }^{+} \mathrm{T}$ cells in the absence of exogenous antigen. J Immunol $2004 ; 173$ : 50-60.

17. Benvenuti F, Hugues S, Walmsley M, et al. Requirement of Racl and Rac2 expression by mature dendritic cells for T cell priming. Science 2004 ; $305: 1150-3$.

18. Faure $S$, Salazar-Fontana LI, Semichon M, et al. ERM proteins regulate cytoskeleton relaxation promoting T cell-APC conjugation. Nat Immunol $2004 ; 5: 272-9$.

19. Depoil D, Zaru R, Guiraud M, et al. Immunological synapses are versatile structures enabling selective T cell polarization. Immunity 2005 ; $22: 185-94$.

20. Yokosuka T, Sakata-Sogawa K, Kobayashi W, et al. Newly generated $T$ cell receptor microclusters initiate and sustain $T$ cell activation by recruitment of Zap70 and SLP-76. Nat Immunol 2005; 6 : 1253-62.

21. Campi G, Varma R, Dustin ML. Actin and agonist MHC-peptide complexdependent T cell receptor microclusters as scaffolds for signaling. J Exp Med $2005 ; 202: 1031-6$.

22. Irvine DJ, Purbhoo MA, Krogsgaard M, Davis MM. Direct observation of ligand recognition by T cells. Nature 2002 ; 419 : 845-9.

23. Lee KH, Dinner AR, Tu C, et al. The immunological synapse balances T cell receptor signaling and degradation. Science 2003 ; 302 : 1218-22.

24. Harriague J, Bismuth G. Imaging antigen-induced PI3K activation in T cells. Nat Immunol $2002 ; 3: 1090-6$.

25. Costello PS, Gallagher M, Cantrell DA. Sustained and dynamic inositol lipid metabolism inside and outside the immunological synapse. Nat Immunol 2002 ; 3 : 1082-9.

26. Fabre S, Lang V, Harriague J, et al. Stable activation of phosphatidylinositol 3-kinase in the T cell immunological synapse stimulates Akt signaling to Fox01 nuclear exclusion and cell growth control. J Immunol 2005 ; 174 : 4161-71.

27. Das V, Nal B, Dujeancourt A, et al. Activation-induced polarized recycling targets $T$ cell antigen receptors to the immunological synapse ; involvement of SNARE complexes. Immunity $2004 ; 20: 577-88$.

28. Huppa JB, Gleimer M, Sumen C, Davis MM. Continuous T cell receptor signaling required for synapse maintenance and full effector potential. Nat Immunol $2003 ; 4: 749-55$.

\section{TIRÉS À PART}

G. Bismuth

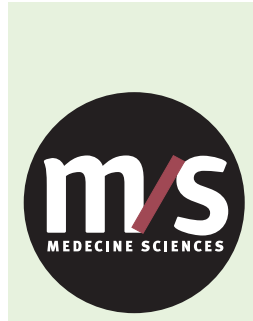

Tarifs d'abonnement M/S - 2006

Abonnez-vous

à Médecine/Sciences
> Depuis 20 ans, grâce à $m / s$, vous vivez en direct les progrès des sciences biologiques et médicales

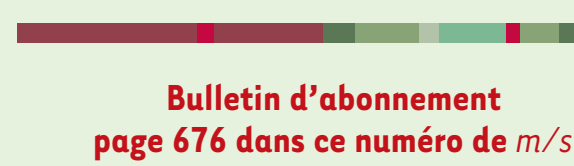

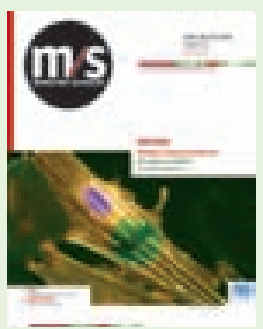

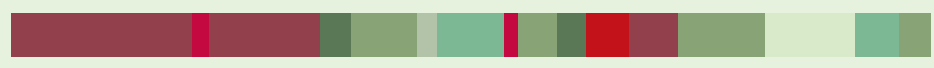

